https://doi.org/10.15407/ujpe64.6.457

I.V. BLONSKYI, ${ }^{1}$ V.M. KADAN, ${ }^{1}$ S.V. PAVLOVA, ${ }^{1}$ I.A. PAVLOV,${ }^{1,2}$

O.I. SHPOTYUK, ${ }^{3,4}$ O.K. KHASANOV ${ }^{5}$

${ }^{1}$ Institute of Physics, Nat. Acad. of Sci. of Ukraine

(46, Nauky Prosp., Kyiv 03028, Ukraine)

${ }^{2}$ Bilkent University, Department of Physics

(Ankara, Turkey)

${ }^{3}$ Vlokh Institute of Physical Optics

(23, Dragomanov Str., Lviv 79005, Ukraine)

${ }^{4}$ Institute of Physics of Jan Dlugosz University

(13/15, Armii Krajowej Al., Czestochowa 42200, Poland)

${ }^{5}$ Scientific-Practical Material Research Centre, NAS of Belarus

(19, Brovki Str., Minsk 220072, Belarus)

\title{
ULTRASHORT LIGHT PULSES
}

IN TRANSPARENT SOLIDS: PROPAGATION

PECULIARITIES AND PRACTICAL APPLICATIONS

\begin{abstract}
The peculiarities of the femtosecond filamentation in Kerr media has been studied using a set of time-resoling experimental techniques. These include the temporal self-compression of a laser pulse in the filamentation mode, repulsive and attractive interactions of filaments, and influence of the birefringence on the filamentation. The propagation of femtosecond laser pulses at the 1550-nm wavelength in $\mathrm{c}$-Si is studied for the first time using methods of time-resolved transmission microscopy. The nonlinear widening of the pulse spectrum due to the Kerr-and plasma-caused self-phase modulation is recorded.

Keywords: femtosecond laser pulses, Kerr effect, femtosecond filaments, crystal silicon, self-focusing, self-phase modulation.
\end{abstract}

\section{Introduction}

One of the main scientific priorities of the XXI-st century is the study of the interaction of femtosecond $(f s)$ laser pulses (of $\sim n \times 10^{-15} \mathrm{~s}$ temporal width) with the matter (see, e.g., [1]). The relevance of such investigations is due to the special properties of $f s$ laser pulses. For example, their ultrashort duration is comparable with the characteristic displacement time of atoms from their equilibrium positions in a molecule, or the transformation time of the crystal grating at

(C) I.V. BLONSKYI, V.M. KADAN, S.V. PAVLOVA,

I.A. PAVLOV, O.I. SHPOTYUK,

O.K. KHASANOV, 2019

ISSN 2071-0194. Ukr. J. Phys. 2019. Vol. 64, No. 6 the structural phase transition. The ultrahigh pulse power and related ultrahigh strength of the electromagnetic field (EMF) produce a gigantic nonlinear polarization of the irradiated medium. The temporal coherence of a spectrally wide $(\sim n \times 10 \mathrm{~nm}) f_{s}$ laser pulse is unusually high, if the laser works in the "comb generator" mode. A characteristic feature of a $f s$ laser pulse propagating in the Kerr medium is the spatio-temporal coupling. It consists in the fact that, in such medium, the EMF of the $f s$ laser pulse cannot be represented as a product of purely spatial and temporal factors. In actual materials, the propagation of such pulses is accompanied by a number of yet insufficiently investigated phenomena of the funda- 
mental nature. Now, these phenomena (formation of $f s$ filaments, generation of a $f s$ supercontinuum with quasiwhite spectrum, conical optical waves, terahertz emission, etc.) are the subject of active studies. The areas of practical use of the $f s$ radiation are wide: microsurgery, LIDARs, pharmacy, precision micromachining of superhard materials, femtosecond laser inscription, etc.

The use of the $f s$ laser sources for scientific and practical purposes requires, as a rule, appropriate methods. Among them, two basic methods are the $f s$ source with quasiwhite spectrum and the precision delay line. The $f s$ pulses with quasiwhite spectrum can be obtained, when the $f s$ laser emission is focused inside some nonlinear optical medium such as water, KTP and BBO crystals, sapphire, etc. However, the time response of electronic detectors, which is limited by the electron mobility in semiconductors, is too slow for the time-resolved studies of ultrafast processes. For that cause, a two-beam pump-probe scheme with precision delay line is needed. The first "pump" pulse of the two-beam scheme excites the object under investigation. The second "probe" pulse, which is controllably delayed from the "pump" with the delay line, "reads out" the relaxation dynamics of the excitation. The principle of such measurements is described in book [2] in more details.

\section{Experimental}

We now briefly report the line of experimental techniques, which we created for the time-resolved studies in the femto-picosecond time domain, including the methods of time-resolved microscopy, and the results of our studies in the fundamental field of filament-induced phenomena and in applications such as the precision $f s$-laser fabrication of optical microelements (microlenses, micromirrors, microwaveguides) in different optical materials (sapphire, quartz, chalcogenide glassy semiconductors, and crystal silicon c-Si).

The following experimental techniques have been developed:

- Two-beam "pump-probe" technique to study the temporal behavior of the light absorption induced by $f s$ laser pulses;

- "Kerr gate" technique for time-resolved investigations of secondary emission spectra with 300 fs temporal resolution;
- Time-resolved microscopic optical polarimetry for the femto-picosecond time domain, which allows recording the spatio-temporal transformation of a light pulse in transparent media;

- Technique of "Z-scan" for the characterization of non-linear refractive indices of transparent materials;

- Technique of temporal compression of fs laser pulses (from140 to $65 \mathrm{fs}$ );

- Methodologies of precision micromachining and micromodification of optical materials for the fabrication of microoptical elements (microwaveguides, microlenses, micromirrors).

\section{Early Studies}

Earlier, we recorded several spectacular phenomena accompanying the formation of $f s$ filaments in transparent Kerr media such as the temporal self-compression of a $f s$ pulse in filaments [3], phase-dependent repulsive and attractive interactions of two intersecting $f s$ filaments [4-6], longitudinal periodicity, which appears in the luminescence of an axial plasma column of filaments in anisotropic crystal media [7].

In [3], the autocorrelator trace of low-energy femtosecond laser pulses with $E_{p}=0.5 \mu \mathrm{J}$, which propagate in a fused silica sample was obtained. We showed that an increase of the pulse energy up to $E_{p}=2 \mu \mathrm{J}$ causes the self-focusing and appearance of a temporally compressed component in the output pulses. The short components of the pulses were extracted using a small axial aperture $2 \mathrm{~mm}$ in diameter, which passes only the temporally compressed axial part of the $\times 200$ magnified output pulse. The temporal width of the autocorrelation function of compressed pulses, $\tau=90 \mathrm{fs}$, is obtained, which corresponds to the 63 -fs duration of the pulse at FWHM. It was shown that the physical cause for the temporal compression is the power dependence of the selffocusing distance, which is described by a Marburger formula [7]. Thus, the laser pulse compressed two and a half times was obtained. It had been used as a probe in the pump-probe measurements having made a more than two-fold improvement of their temporal resolution to be possible.

The interaction between two filaments in air and transparent solids [8-10] have attracted a considerable interest of researchers, because the understanding of this process is important for the control over the multiple filamentation during a laser modification

ISSN 2071-0194. Ukr. J. Phys. 2019. Vol. 64, No. 6 


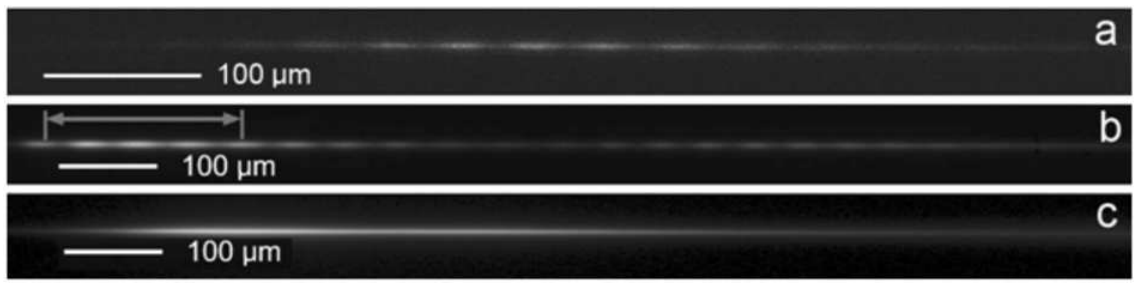

Fig. 1. Filaments in crystal quartz $(a)$ and sapphire $(b, c)$. Input beam is plane-polarized at $45^{\circ}$ to the crystal $c$-axis $(a-c)$. Input beam is plane-polarized at $90^{\circ}$ to the crystal $c$-axis $(c)$. The input pulse energy $E_{i n}=0.88 \mu \mathrm{J}$, central wavelength $\lambda_{m}=800 \mathrm{~nm}$, pulse duration $\tau_{p}=150 \mathrm{fs}(a, b, d)$. The pulse travels from left to right in all cases (after [7])
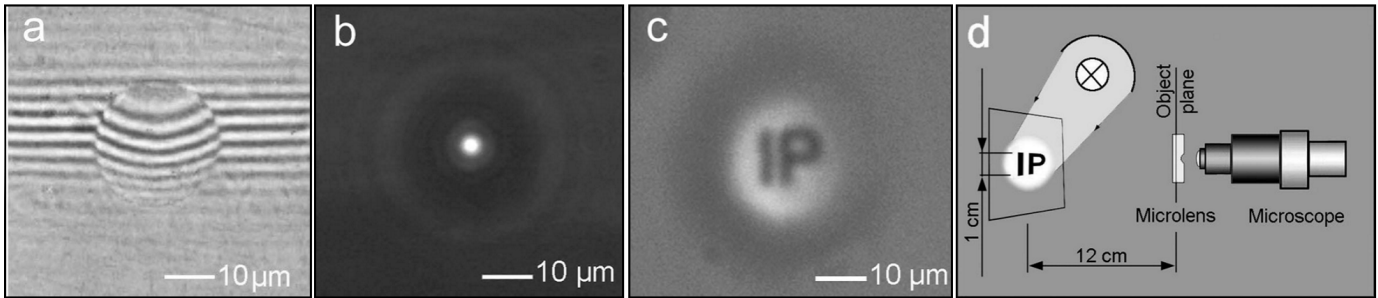

Fig. 2. Microinterferogram of the microlens formed on the surface of a sample $\mathrm{S}$ after the single-pulse exposure $\left(E_{p}==12 \mu \mathrm{J}, \delta=0\right)(a)$. The virtual focal spot of the microlens at the depth of $140 \mu \mathrm{m}$ beneath the surface of the sample under the LED illumination at $\lambda=0.63 \mu \mathrm{m}(b)$. The virtual image of the letters "IP" formed by the microlens $(c)$. The measurement geometry $(d)$ (after [12])

of optoelectronic materials. Changing the phase difference between two mutually coherent femtosecond laser pulses coinciding in time, we have demonstrated the control over the interaction between two intersecting filaments. We observed the "attraction" followed by the "fusion" of in-phase and the "repulsion" of antiphase filaments in [4-6]. Note that the anisotropy of the sapphire crystal has little effect on the filamentation in this case, because both beams had been directed at a small angle of $2.4^{\circ}$ to the crystal $c$-axis.

The picture of the femtosecond filamentation drastically changes in the case of anisotropic propagation geometry of the beam. In [7], we observed, for the first time, the phenomenon of longitudinally periodic filamentation of the $f s$ laser radiation propagating in positive $\left(n_{0}<n_{e}\right.$; crystalline quartz) and negative $\left(n_{0}>n_{e}\right.$; sapphire) birefringent crystalline media (Fig. 1). We also showed that the observed longitudinal periodicity of a filament is caused by the periodic change in the polarization of a pulse traveling in the birefringent medium in combination with the cross-sectional difference in the multiphoton absorptions for linear and circular polarizations.

Earlier, we focused on more practical aspects of the interaction of the $f s$ laser pulses with transpar-

ISSN 2071-0194. Ukr. J. Phys. 2019. Vol. 64, No. 6 ent materials. Using a femtosecond laser inscription, we produced microwaveguides in chalcogenide glasses [11]. In [12], we demonstrated a new maskless process of production of diffraction-limited microlenses and micromirrors in a chalcohalide glass composed of $65 \% \mathrm{GeS}_{2}, 25 \% \mathrm{Ga}_{2} \mathrm{~S}_{3}$, and $10 \% \mathrm{CsCl}$ using a single $f s$ laser pulse. Thus, programming the scanning sequence of the laser beam, arbitrarily complex geometry of the lens array can be produced. Figure 2 shows some characterization results of the obtained microlenses.

\section{Spatio-Temporal Transformation of $f s$ Laser Pulses at $1550 \mathrm{~nm}$ in c-Si}

Next, we go to the latest original results on the phenomena accompanying the propagation of IR femtosecond laser pulses in crystal silicon (c-Si), which is the important material of electronics and photonics. Like in other photonic materials, a precise micromodification of optical properties of c-Si can be achieved with the use of $f s$ laser pulses. Given that photonic devices for light guiding, splitting, and modulation are very much needed in integrated Si photonics, a fs laser with longer wavelength corresponding 

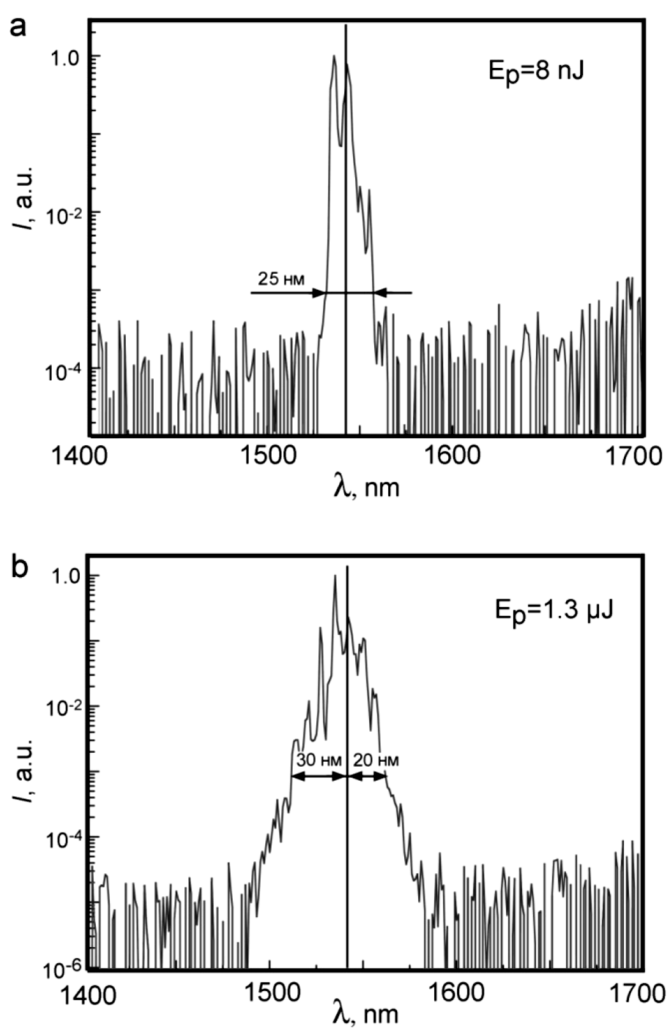

Fig. 3. Spectra of the output laser pulses, which passed a 0.5mm-thick c-Si plate at two different input pulse energies $E_{p}$. Linear regime $(a)$, nonlinear regime $(b)$

to the c-Si transparency region should be used for its bulk modification.

In the next experiments, we used a selfmade erbium-ytterbium fiber laser as an excitation source. The laser consists of an oscillator and two amplifiers. It produces pulses with central wavelength $\lambda_{0}=1.55 \mu \mathrm{m}, 0.4-0.45$-ps time width, and repetition rate up to $1 \mathrm{MHz}$. The average output power is $1.3 \mathrm{~W}$. Using a new experimental technique of timeresolved pump-probe microscopy, we have shown that the tightly focused $1550-\mathrm{nm}$ femtosecond radiation can permanently modify the refractive index of c$\mathrm{Si}$, thus creating a built-in microwaveguide capable of guiding and transporting light in the IR range [13]. Apart from this, a nonlinear spatial-temporal transformation of the fs laser pulses at $1.5 \mu \mathrm{m}$ was observed in c-Si, which results both in the filamentation and a change of the frequency spectrum of pulses. First, we recorded an appreciable spectral widening of the output high-energy laser pulses in comparison with low-energy laser ones at $1.5 \mu \mathrm{m}$ wavelength with the repetition rate $f=250 \mathrm{kHz}$, and the temporal width $\tau_{p}=450 \mathrm{fs}$, which have been focused into a 0.5 -mm-thick c-Si plate with an aspherical lens of the $35-\mathrm{mm}$ focal distance. The measured spectra of the output laser pulses at two different input pulse energies are shown on the logarithmic scale in Fig. 3, a (single pulse energy $E_{p}=8 \mathrm{~nJ}$ ) and Fig. 3 , $b$, (single pulse energy $\left.E_{p}=1.3 \mu \mathrm{J}\right)$. Considering that the self-focusing critical power $P_{\text {crit }}$ [14] of laser pulses at the $1.5-\mu \mathrm{m}$ wavelength with the temporal width $\tau_{p}=450 \mathrm{fs}$ in c-Si corresponds to the pulse energy $E_{p}=12 \mathrm{~nJ}[15]$, we conclude that the propagation regime in Fig. $3, a$ is linear with $P<P_{\text {crit }}$, while, in Fig. 3, b, it is highly nonlinear with $P \sim$ $\sim 100 P_{\text {crit }}$. Several peculiar features of the spectra in Fig. 3 should be noted. The first is a significant reshaping of the output spectrum in a nonlinear mode, which is more pronounced at the low-intensity band wings, while the spectral widening around the central frequency is weaker. So, the band width increases two-fold from 25 to $50 \mathrm{~nm}$ at the level $10^{-3}$. This can be attributed to the nonlinear character of the self-phase modulation (SPM). Indeed, the almost instantaneous nonlinear change of the refractive index by the Kerr mechanism ( $\sim$-fs response time), which causes the spatial self-focusing of the light pulse, also changes its phase $\phi$, thus generating new frequencies [14]. If the refractive index $n$ depends on the light intensity $I$, then, in the case of a flat wave,

$\varphi=\frac{n_{0} \omega_{0}}{c} z+n_{2} I(t) \frac{\omega_{0}}{c}-\omega_{0} t$,

and new frequencies appear:

$\omega(t)=-\frac{\partial \varphi}{\partial t}=\omega_{0}-\frac{n_{2} \omega_{0} z}{c} \frac{\partial I(t)}{\partial t}$

So, the intensity change generates new frequencies. If $n_{2}>0$, the rising edge of the pulse with $\frac{\partial I}{\partial t} \geqslant 0$ causes the red shift, while blue-shifted frequencies are generated at the pulse rear-end. The symmetric (e.g., Gaussian) temporal shape of a pulse results in symmetric blue and red frequency shifts. Apart from the Kerr mechanism, the appearance of a plasma generated by the two-photon absorption also causes the SPM, because it changes the refractive index. If the plasma density $N_{e}$ is significantly below the critical value $N_{\mathrm{cr}}$, then it decreases the refractive index:

$\Delta n \approx-\frac{N_{e}}{2 n_{0} N_{\mathrm{cr}}}$.

ISSN 2071-0194. Ukr. J. Phys. 2019. Vol. 64, No. 6 
In such a case, the plasma-induced shift $\Delta \omega$ of the pulse central frequency $\omega_{0}$ is

$\Delta \omega(t)=\frac{-\partial \phi}{\partial t} \approx \frac{\omega_{0} z}{2 c n_{0} N_{\mathrm{cr}}} \frac{\partial N_{e}(t)}{\partial t}$,

thereby shifting the pulse frequency to the blue side [16]. So, due to a nonlinear character of the SPM, the frequency changes not uniformly over the whole area of the pulse cross-section, but predominantly in the paraxial volume of the pulse, which contains only a part of the total pulse energy. In simplified terms, the total spectrum of the output pulse is formed by a sum of the spectrally shifted contribution of the axial part and the almost unchanged contribution of the peripheral part of the pulse. As a result, the widening of the total spectrum becomes more pronounced at its wings. In distinct from the Kerr-caused shift, the plasma-caused SPM shifts the spectrum only to the blue side. Thus, the asymmetry of the output spectrum in Fig. 3, b, clearly indicates the participation of the laser-induced plasma in SPM. In comparison with the linear spectrum in Fig. 3, $a$, the shift at the level of $10^{-3}$ is $17.5 \mathrm{~nm}$ to the blue side and only $7.5 \mathrm{~nm}$ to the red one in the spectrum in Fig. 3, b. Assuming the symmetric blue and red Kerr shifts of $7.5 \mathrm{~nm}$, we conclude that the plasma-caused SPM gives an additional $10 \mathrm{~nm}$ of the blue wavelength shift in the present experiment, thus exceeding the Kerr contribution.

The observed SPM-caused spectral widening clearly suggests the presence of nonlinear temporal dynamics in the propagating pulse. However, no temporal dynamics of laser pulses at $1.5 \mu \mathrm{m}$ wavelength with $\tau_{p}=100$ fs $\left(P \sim 24 P_{\text {crit }}\right)$, focused by a $f=100 \mathrm{~mm}$ lens into a $8 \mathrm{~mm}$-thick c-Si plate was recorded in [15]. We believe that the observed spectral broadening does not contradict the conclusion about the absence of a temporal dynamics in [15]. First, in [15], the pulse power is 4 times lower. Second, we believe that, apart from the looser focusing and a smaller power excess over $P_{\text {crit }}$, the main cause for the discrepancy is that we looked for different manifestations of the temporal dynamics. In [15], the authors looked for a change in the temporal width of the output pulse using the autocorrelation measurements. Here, we measured the small changes in the spectral width of the output pulse. Thus, in our experiment, the temporal dynamics does manifest itself in the frequency domain, but not in the temporal reshaping of the pulse envelope.

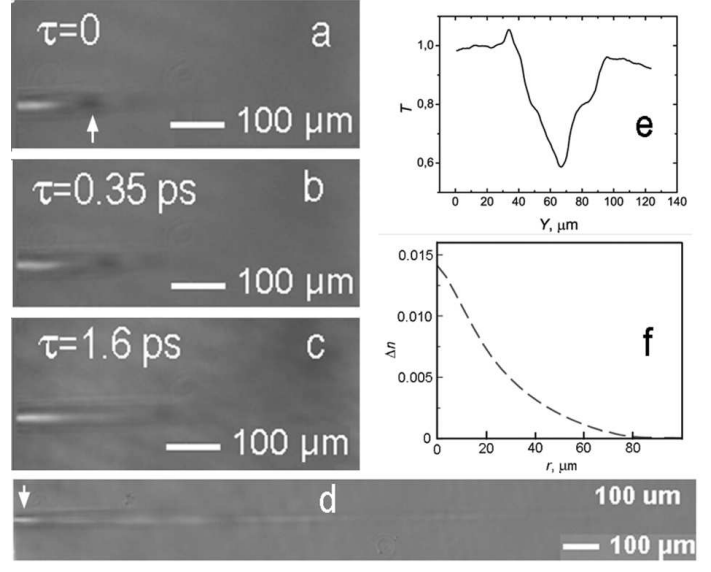

Fig. 4. Transmission pictures of the development of the plasma channel in c-Si $(a-c)$ in a 0.5 -mm-thick c-Si specimen under the fs excitation at the $1550-\mathrm{nm}$ wavelength at the 200$\mu \mathrm{m}$ defocusing. Steady-state plasma channel at $\tau=100 \mathrm{ps}$ and $100 \mu \mathrm{m}$ defocusing $(d)$. The TPA absorption profile $(e)$ is taken at the cross-section indicated by an upward white arrow in $(a)$. The refractive index profile $(f)$ is retrieved from the transmission profile taken at the cross-section, indicated by a downward white arrow in $(d)$

To confirm the presence of a plasma trail, which follows the fs laser pulse propagating in s-Si, we performed the following experiment. We used an erbium-ytterbium fiber laser described above as an excitation source. Using a new experimental technique of time-resolved pump-probe microscopy, we have obtained the instantaneous shadowgraphs of $f s$ laser pulses with the repetition rate $f=250 \mathrm{kHz}$ and the single pulse energy $E_{p}=1.14 \mu \mathrm{J}$, propagating in a c-Si plate. The laser beam was focused by an aspherical lens with 35-mm focal distance onto a c-Si plate in parallel with its plane through its 0.5 -mm-thick side face. The shadowgraphs of the pump pulse (Fig. 4, $a^{-}$ $c$ ) were obtained in the transmission geometry at different time delays of a probe pulse, which was split-off from the same laser beam, viewing the propagation area with a microscope provided with an IR In-GaAs camera. The shadowgraph in Fig. $4, d$ is taken at $\tau=100$ ps for the steady-state plasma channel, after the pump pulse have already passed the viewing area.

To the best of our knowledge, in this work we have used, for the first time, time-resolved transmission microscopy to observe the propagation of IR light pulses at the $1550-\mathrm{nm}$ wavelength in silicon. Thus, the stages of spatial transformation and the complex shape of a TPA-induced plasma column have 
been revealed. The dark spot in Fig. $4, a-c$ shifting to the right, when $\tau$ is increasing, is, in fact, the laser pulse itself, which is visualized due to the TPA involving one pump and one probe photons. Note that the calculated propagation velocity of the observed TPA spot equals to that of the light velocity in c-Si, and its width along the propagation axis is consistent with the laser pulse duration $\tau_{p}=450 \mathrm{fs}$ with regard for the refractive index of $\mathrm{c}-\mathrm{Si} n=3.5$ at the $1.5-\mu \mathrm{m}$ wavelength. This is because of the fact that the response time of the TPA process is extremely short. A nonresonant electronic transition, as follows from the uncertainty principle, occurs on a time scale of $\left|\omega-E_{g} / h\right|^{-1}$, which is less than 10 fs for the frequencies well below the band gap [17]. Using the above-mentioned pulse parameters and assuming the diffraction-limited diameter of the laser focal spot $d=24 \mu \mathrm{m}$, we estimated the transient absorption induced by the pump pulse through the TPA process. The coefficient of TPA is taken as $\beta_{\mathrm{TPA}}=$ $=4 \times 10^{-6} \mu \mathrm{m} / \mathrm{W}[18]$. According to the estimation, the presence of the above laser pulse induces the linear absorption value $\alpha_{\text {ind }}=\beta_{\mathrm{TPA}} I=4.5 \times$ $\times 10^{-2} \mu \mathrm{m}^{-1}$ in $\mathrm{c}-\mathrm{Si}$, which agrees well with the measured absorption profile (Fig. 4,e).

The trail, which follows the propagating laser pulse in Fig. $4, c-d$, is formed by a laser-induced plasma generated in the TPA processes involving two pump photons. As can be seen from formula (3), the plasma gives a negative change in the refractive index, which acts as a concave cylindrical lens. This enables the observation of the plasma column using the light microscopy. In Fig. 4, $a-c$, the object plane of the microscopic objective is shifted by $200 \mu \mathrm{m}$ beneath the actual plasma column, so the virtual focus of the concave plasma lens is seen as a light axial line surrounded with darker areas. The image of the plasma column almost disappears, when it lies exactly in the object plane, indicating a much lower plasma absorption in comparison with TPA. Increasing $\tau$, we have observed that the image of the plasma column keeps unchanged for several ns (Fig. 3, d), in agreement with the free carrier lifetime of more than $10 \mathrm{~ns}$ in c-Si, but completely disappears, if the pump light is turned off.

To retrieve the profile of a plasma-caused change in the refractive index $\Delta n$ from the defocused microscopic image in Fig. 4, $d$, we have used the same procedure, as described in [13], having applied the transport-of-intensity equation and the inverse Abel transformation to the intensity profile of the plasma column at the position indicated by the white arrow. As can be seen from the retrieved profile of $\Delta n$ in Fig. $4, f$, the resulting plasma-caused decrease in the refractive index reaches $\sim 0.014$ on the axis. Further, to calculate the plasma density $N_{e}$ from Eq. (3), we should know the critical plasma density $N_{\text {cr }}$. It could be found from the formula for the plasma frequency $\omega_{p}=\left(4 \pi N_{e} e^{2} / \mu\right)^{1 / 2}$, assuming that $\omega_{p}=\omega_{0}$. Note that the effective mass $\mu$ in the formula for the plasma frequency in c-Si depends on $N_{e}$. According to [19], in the case of low density plasma, $\mu=0.12 m_{e}$, while the heavy excitation $\left(N_{e} \sim 10^{21} \mathrm{~cm}^{-1}\right)$ changes the density of states, resulting in the increased $\mu=0.5 m_{e}$ [20]. Assuming $\mu=0.12 m_{e}$ and using the retrieved value $\Delta n=0.014$, we get from formula (3) that the plasma density $N_{e}=5.7 \times 10^{18} \mathrm{~cm}^{-3}$, while $\mu=0.5 m_{e}$ results in $N_{e}=2.4 \times 10^{19} \mathrm{~cm}^{-3}$.

Now, we compare this $N_{e}$ value with that calculated from the pulse parameters and the TPA coefficient. Indeed, assuming $\beta_{\mathrm{TPA}}=4 \times 10^{-6} \mu \mathrm{m} / \mathrm{W}$, pulse energy $=1.14 \mu \mathrm{J}$ (for $\tau_{p}=450 \mathrm{fs}$ ), and the diameter of the laser focal spot $d=24 \mu \mathrm{m}$ and taking into account that the energy of a photon pair needed to generate the e-h pair in TPA $E_{e-h}=2.56 \times 10^{-19} \mathrm{~J}$, we estimate the number of free electrons per $1 \mathrm{~cm}^{3}$ created by one laser pulse, as $N_{e}=E_{p} / E_{e-h}=1.3 \times 10^{20} \mathrm{~cm}^{-3}$, which exceeds the above-mentioned value $N_{e}=2.4 \times 10^{19} \mathrm{~cm}^{-3}$ estimated from the microscopic image by more than half the order of magnitude. In our opinion, the difference can arise from the fact that TPA attenuates and plasma defocuses the pump pulse even before it reaches the focal spot. As a result, the $N_{e}$ value calculated for a not attenuated pulse occurs overestimated [21].

The tighter beam focusing with a lens of $9-\mathrm{mm}$ focal distance causes irreversible changes in the refractive index of the material at the focus. In this way, we created permanent waveguides deeply built-in in the bulk of c-Si, which are promising for the use in integrated silicon photonics [13].

In summary, we have recorded, for the first time, a spatio-temporal transformation of 450-fs-long femtosecond laser pulses with the pulse power up to $1.3 \mu \mathrm{J}$ at the $1550-\mathrm{nm}$ wavelength in c-Si using timeresolved defocusing microscopy. The propagating $f_{s}$ laser pulse leaves the column of a long-living TPA- 
induced plasma on its trail, which reduces the refractive index by the value of $\Delta n=0.014$. The generation of new frequencies in the propagating pulse is recorded, which manifests itself as a widening of the pulse spectrum due to SPM. A stronger frequency shift to the blue side is attributed to the SPM by a laser-induced plasma.

This research was performed using the experimental equipment of the Laser Femtosecond Center for Collaborative Use of the National Academy of Sciences of Ukraine. We acknowledge the support from the NAS of Ukraine (project BC/201).

1. C. Rullière. Femtosecond Laser Pulses. Principles and Experiments (Springer, 1998).

2. I.V. Blonskyi, V.M. Kadan. Ultrafast and Superpowerful Light Pulses in Condensed Media (Naukova Dumka, 2017) (in Ukrainian).

3. I.V. Blonskyi, V.M. Kadan, V.M. Puzikov, L.A. Grin'. Temporal autolocalization of femtosecond light pulses in the filaments observed in fused silica. Ukr. J. Phys. Opt. 14, 85 (2013).

4. A.A. Dergachev, V.N. Kadan, S.A. Shlenov. Interaction of noncollinear femtosecond laser filaments in sapphire. Quantum Electron. 42, 125 (2012).

5. I. Blonskyi, V. Kadan, O. Shpotyuk et al. Filamentation in the intersection region of two femtosecond laser beams in sapphire. Ukr. J. Phys. 59, 344 (2014).

6. I. Blonskyi, V. Kadan, O. Shpotyuk et al. Interaction of femtosecond filaments in sapphire. Proc. of SPIE $\mathbf{7 9 9 3 ,}$ 79931C-1 (2011).

7. I. Blonskyi, V. Kadan, Y. Shynkarenko et al. Periodic femtosecond filamentation in birefringent media. Applied Phys. B 120, 905 (2015).

8. A.C. Bernstein, M. McCormic, G.M. Dyer et al. Two-beam coupling between filament-forming beams in air. Phys. Rev. Lett. 102, 123902 (2009).

9. S. Tzortzakis, L. Berge, A. Couairon et al. Breakup and fusion of self-guided femtosecond light pulses in air. Phys. Rev. Lett. 86, 5470 (2001).

10. T. Xi, X. Lu, J. Zhang. Interaction of light filaments generated by femtosecond laser pulses in air. Phys. Rev. Lett. 96, 025003 (2006).

11. I. Blonskyi, V. Kadan, O. Shpotyuk et al. Filamentinduced self-written waveguides in glassy $\mathrm{As}_{4} \mathrm{Ge}_{30} \mathrm{~S}_{66}$. Appl. Phys. B 104, 951 (2011).
12. V. Kadan, I. Blonskyi, Y. Shynkarenko et al. Single-pulse femtosecond laser fabrication of concave microlens and micromirror arrays in chalcohalide glass. Optics and Laser Technology 96 , 283 (2017).

13. I. Pavlov, O. Tokel, S. Pavlova, V. Kadan et al. Femtosecond laser written waveguides deep inside silicon. Opics Letters 42, 3028 (2017).

14. Y.R. Shen. The Principles of Nonlinear Optics (Wiley, 1984).

15. D. Faccio, M. Clerici, A. Averchi et al. Kerr-induced spontaneous Bessel beam formation in the regime of strong twophoton absorption. Opt. Express 16, 8213 (2008).

16. B.M. Penetrante, J.N. Bardsley, W.M. Wood et al. Ionization-induced frequency shifts in intense femtosecond laser pulses. J. Opt. Soc. Am. B 9, (2032) 1992.

17. Q. Lin, O.J. Painter, G.P. Agrawal. Nonlinear optical phenomena in silicon waveguides: Modeling and applications. Opt. Express 15, 16604 (2007).

18. J.F. Reintjes, J.C. McGroddy. Indirect two-photon transitions in Si at $1.06 \mu \mathrm{m}$. Phys. Rev. Lett. 30, 901 (1973).

19. M. Combescot, J. Bok. Electron-hole plasma generation and evolution in semiconductors. J. Lumin. 30, 1 (1985).

20. J.R. Chelikowsky, M.R. Cohen. Nonlocal pseudopotential calculations for the electronic structure of eleven diamond and zinc-blende semiconductors. Phys. Rev. B 14, 556 (1976).

21. V.V. Kononenko, E.V. Zavedeev, V.M. Gololobov. The effect of light-induced plasma on propagation of intense fs laser radiation in c-Si. Appl. Phys. A 122, 293 (2016).

Received 21.11.17

I.В. Блонсъкий, В.М. Кадан, С.В. Павлова,

І.А. Павлов, О.Й. Шпотюк, О.Х. Хасанов

УЛЬТРАКОРОТКІ СВІТЛОВІ ІМПУЛЬСИ

В ПРОЗОРИХ ТВЕРДИХ ТІЛАХ: ОСОБЛИВОСТІ

ПОШИРЕННЯ ТА ПРАКТИЧНІ ЗАСТОСУВАННЯ

$\mathrm{P}$ е $з$ ю м е

В керрівських середовищах часороздільними експериментальними методами вивчено особливості фемтосекундної філаментації, зокрема такі, як часове самостиснення лазерного імпульсу в режимі філаментації, відштовхування та тяжіння філаментів, а також вплив двопроменезаломлення на філаментацію. Поширення фемтосекундних лазерних імпульсів на довжині хвилі 1550 нм в c-Si вперше вивчено методом часороздільної мікроскопії пропускання світла. Виявлено нелінійне розширення спектра імпульсів внаслідок керрівської та плазмової фазової самомодуляції. 